\title{
Gluon Condensate and Non-Perturbative Quark-Photon Vertex *
}

\author{
Munshi G. Mustafa ${ }^{1 \dagger}$, Andreas Schäfer ${ }^{2}$ and Markus H. Thoma ${ }^{3 \ddagger}$ \\ ${ }^{1}$ Institut für Theoretische Physik, Universität Giessen, 35392 Giessen, Germany \\ ${ }^{2}$ Institut für Theoretische Physik, Universität Regensburg, 93040 Regensburg, Germany \\ ${ }^{3}$ Theory Division, CERN, CH-1211 Geneva 23, Switzerland
}

(April 2, 2018)

\begin{abstract}
We evaluate the quark-photon vertex non-perturbatively taking into account the gluon condensate at finite temperature. This vertex is related to the previously derived effective quark propagator by a QED like Ward-Takahashi identity. The importance of the effective vertex for the dilepton production rate from a quark-gluon plasma is stressed.
\end{abstract}

PACS numbers: 12.38.Lg

Typeset using REVTEX

*Supported by BMBF, GSI Darmstadt, DFG, and Humboldt foundation

${ }^{\dagger}$ Humboldt fellow and on leave of absence from Saha Institute of Nuclear Physics, 1/AF Bidhan Nagar, Calcutta 700 064, India

${ }_{\ddagger}^{\ddagger}$ Heisenberg fellow 
During the last two decades a substantial amount of activity, both experimentally and theoretically, has been devoted to an improved understanding of the so called quark-gluon plasma (QGP). The prime goal is to find a reliable signature for this new phase, which in turn urgently requires a better theoretical understanding. Some insight can be gained within the framework of thermal field theory [1]. Within the perturbative approach the thermal effects are known to substantially alter soft modes, changing the dispersion relations, and allowing for Landau damping as well as for new modes of propagation for both fermionic and bosonic quasi-particles [2]. However, perturbative calculations based on bare propagators and vertices lead to gauge dependent and infrared divergent results. This inconsistency is due to the fact that at high temperature higher order loop diagrams can contribute to lower order in the coupling constant. This problem has been cured to some extent by the Hard Thermal Loop (HTL) resummation technique developed by Braaten and Pisarski [3], and much progress has also been achieved within HTL resummed perturbation theory in describing dynamical properties of the QGP. In one particular application, the dilepton rate, obtained by using HTL resummed propagators and vertices [1, was e.g. shown to exhibit sharp structures in the low invariant mass regime caused by the presence of collective quark modes in the medium. However, it seems that quite generally non-perturbative effects [5] dominate in the temperature regime attainable in heavy-ion experiments.

Such non-perturbative effects were made explicit by lattice QCD calculations of e.g., effective parton masses [6], hadronic correlators [7], and the gluon condensate [5], below and above the phase transition temperature. So far it is beyond the scope of lattice calculations to compute dynamical quantities. Gluon and quark condensates, however, which describe the non-perturbative imprints of the QCD ground state, have extensively been used in QCD sum rules [8] for studying hadron properties at zero and finite temperature. At zero temperature the quark propagator containing the gluon condensate has been constructed by Lavelle and Schaden [9]. This propagator has been used in a number of investigations [10]. Recently, as an alternative method to lattice and perturbative QCD, it has been suggested in Ref. [11] to include the temperature-dependent gluon condensate into parton propagators at finite temperature. In this way non-perturbative effects can be taken into account within the Greens function technique to compute static [12] as well as dynamic quantities [13]. In the latter work the dilepton production rate, which represents a promising signature for the QGP formation in relativistic heavy-ion collisions, has been calculated using an effective quark propagator containing the gluon condensate. Presently such calculations are, however, not yet self-consistent. One has to introduce in addition an effective quark-photon vertex, which is related to the effective quark propagator by the QED Ward-Takahashi identity. As a first step we intend to calculate the effective quark-photon vertex in the presence of the gluon condensate, both at zero and finite temperature, and to show that it fulfills the Ward identity. In a later step we plan to repeat the dilepton calculation with this vertex, which is, however, a highly non-trivial task in view of the complex form of the result we are going to present.

The effective vertex, containing the effects of the gluon condensate in leading order, is given by Fig. 1 and can be written as

$$
\Gamma^{\nu}\left(P_{1}, P_{2}\right)=\Gamma_{0}^{\nu}+\Gamma_{G}^{\nu}\left(P_{1}, P_{2}\right)
$$

where the bare vertex is given by $\Gamma_{0}^{\nu}=-i e \gamma^{\nu}$ with the usual QED coupling constant $e$. 
For massless bare quarks the one loop correction containing the gluon condensate can be written, using standard Feynman rules, as

$$
\Gamma_{G}^{\nu}\left(P_{1}, P_{2}\right)=-\frac{4}{3} g^{2} e \int \frac{\mathrm{d}^{4} K}{(2 \pi)^{4}} \frac{\left[\gamma^{\mu}\left(\not P_{2}+\not K\right) \gamma^{\nu}\left(\not P_{1}-\not K\right) \gamma^{\rho}\right]}{\left(P_{1}-K\right)^{2}\left(P_{2}+K\right)^{2}} \tilde{D}_{\mu \rho}(K),
$$

where $g^{2}=4 \pi \alpha_{s}$ is the strong coupling constant and the four momentum is defined as $Q \equiv\left(q_{0}, \vec{q}\right)$ and $q=|\vec{q}|$. The general expression for the non-perturbative gluon propagator containing the gluon condensate is given by 9

$$
\tilde{D}_{\mu \rho}(K)=D_{\mu \rho}^{\text {full }}(K)-D_{\mu \rho}^{\text {pert }}(K)
$$

where the subtraction of the perturbative propagator guarantees that $\tilde{D}_{\mu \rho}(K)$ is gauge independent.

The effective quark propagator containing the gluon condensate is given in Fig. 2 and can be written as

$$
S^{-1}(P)=S_{0}^{-1}(P)-\Sigma_{G}(P)
$$

where the quark self energy for massless bare quarks reads

$$
\Sigma_{G}(P)=\frac{4}{3} i g^{2} \int \frac{\mathrm{d}^{4} K}{(2 \pi)^{4}} \tilde{D}_{\mu \rho}(K) \gamma^{\mu} \frac{\not P-\not K}{(P-K)^{2}} \gamma^{\rho} .
$$

So far we have discussed general expressions for the vertex and propagator containing the gluon condensate. Next we would like to obtain the zero temperature vertex related to the quark propagator by the Ward-Takahashi identity.

At zero temperature, transversality and Lorentz-invariance demand that the gluon propagator has the following form

$$
\tilde{D}_{\mu \rho}(K)=\left[g_{\mu \rho}-\frac{K_{\mu} K_{\rho}}{K^{2}}\right] \tilde{D}\left(K^{2}\right)
$$

The lowest moments of the function $\tilde{D}\left(K^{2}\right)$ of the gluon propagator in (6) are related to the gluon condensate by 9

$$
\left\langle: G^{2}:\right\rangle=\left\langle: G_{a}^{\mu \rho}(0) G_{\mu \rho}^{a}(0):\right\rangle=48 i \int \frac{\mathrm{d}^{4} K}{(2 \pi)^{4}} K^{2} \tilde{D}\left(K^{2}\right),
$$

where $a$ is the color index of the $\mathrm{SU}(3)$ color gauge group and the gluon field strengths are summed over color.

Following Ref. 9] we expand the product of the quark propagators in (2) for small loop momentum $K$. Keeping terms which are at most bilinear in $K$ leads to

$$
\begin{aligned}
\frac{1}{\left(P_{1}-K\right)^{2}\left(P_{2}+K\right)^{2} \approx \frac{1}{P_{1}^{2} P_{2}^{2}}}[ & 1+2 \frac{K \cdot P_{1}}{P_{1}^{2}}-2 \frac{K \cdot P_{2}}{P_{2}^{2}}-\frac{K^{2}}{P_{1}^{2}}-\frac{K^{2}}{P_{2}^{2}}+4 \frac{\left(K \cdot P_{1}\right)^{2}}{P_{1}^{4}} \\
& \left.+4 \frac{\left(K \cdot P_{2}\right)^{2}}{P_{2}^{4}}-4 \frac{\left(K \cdot P_{1}\right)\left(K \cdot P_{2}\right)}{P_{1}^{2} P_{2}^{2}}\right] .
\end{aligned}
$$


Substituting (6) and (8) into (2), keeping terms only bilinear in $K$, and using (7) we obtain the following expression for the one-loop correction of the vertex:

$$
\begin{gathered}
\Gamma_{G}^{\nu}\left(P_{1}, P_{2}\right)=\frac{i e g^{2}}{36 P_{1}^{2} P_{2}^{2}}\left[\gamma^{\nu}+\frac{4}{3}\left(P_{1}+P_{2}\right)^{2} \frac{P_{1}^{\nu} P_{2}}{P_{1}^{2} P_{2}^{2}}-\frac{2}{3} \gamma^{\nu}\left(P_{1}+P_{2}\right)^{2} \frac{P_{1} P_{2}}{P_{1}^{2} P_{2}^{2}}+\frac{1}{3} \frac{P_{1}^{\nu} P_{2}}{P_{2}^{2}}+\frac{1}{3} \frac{P_{2}^{\nu} P_{1}}{P_{1}^{2}}\right. \\
\left.-\frac{5}{3} \frac{P_{2}^{\nu} P_{2}}{P_{2}^{2}}-\frac{5}{3} \frac{P_{1}^{\nu} P_{1}}{P_{1}^{2}}\right]\left\langle: G^{2}:\right\rangle
\end{gathered}
$$

Following the same procedure, the quark self energy in (5) becomes [9]

$$
\Sigma_{G}(P)=g^{2} \frac{\not P}{P^{4}} \frac{\left\langle: G^{2}:\right\rangle}{36}
$$

Here we would like to point out that at zero temperature the quark self energy should also have a contribution containing the quark condensate, which complicates the problem substantially. However, we are interested in the case $T>T_{c}$ and in the deconfining phase the quark condensate is zero.

Combining (11) and (9) it is now easy to show that the effective vertex, containing the non-perturbative gluon condensate, satisfies indeed the Ward-Takahashi identity

$$
\begin{aligned}
\left(P_{1}+P_{2}\right)_{\nu} \Gamma^{\nu}\left(P_{1}, P_{2}\right) & =-i e\left[\left(\not P_{1}-\Sigma_{G}\left(P_{1}\right)\right)-\left(-\not P_{2}-\Sigma_{G}\left(-P_{2}\right)\right)\right] \\
& =-i e\left[S^{-1}\left(P_{1}\right)-S^{-1}\left(-P_{2}\right)\right]
\end{aligned}
$$

which relates the vertex to the effective quark propagator, (4) and (10).

In the following we evaluate the vertex at finite temperature. As usually for finite temperature a complication arises due to the fact that temperature is defined in a fixed reference frame and Lorentz invariance is lost. Therefore the non-perturbative gluon propagator in (3) has a different form for longitudinal and transverse gluons. It can be written as [14]

$$
\tilde{D}_{\mu \rho}(K)=P_{\mu \rho}^{l} \tilde{D}_{l}(K)+P_{\mu \rho}^{t} \tilde{D}_{t}(K)
$$

where the longitudinal and transverse projection tensors are given as

$$
\begin{aligned}
& P_{\mu \rho}^{t}+P_{\mu \rho}^{l}=\frac{K_{\mu} K_{\rho}}{K^{2}}-g_{\mu \rho} \\
& P_{\mu 0}^{t}=0, \quad P_{i j}^{t}=\delta_{i j}-\frac{k_{i} k_{j}}{k^{2}} .
\end{aligned}
$$

In the rest frame of the heat bath the fermion self energy for vanishing bare mass can be written as 15

$$
\Sigma_{G}(P)=-a\left(p_{0}, p\right) \not P-b\left(p_{0}, p\right) \gamma_{0}
$$

with the scalar functions

$$
\begin{aligned}
& a\left(p_{0}, p\right)=\frac{1}{4 p^{2}}\left[\operatorname{Tr}\left(\not P \Sigma_{G}\right)-p_{0} \operatorname{Tr}\left(\gamma_{0} \Sigma_{G}\right)\right], \\
& b\left(p_{0}, p\right)=\frac{1}{4 p^{2}}\left[P^{2} \operatorname{Tr}\left(\gamma_{0} \Sigma_{G}\right)-p_{0} \operatorname{Tr}\left(\not P \Sigma_{G}\right)\right],
\end{aligned}
$$


and $\Sigma_{G}$ as given by $(5)$.

Using (12) and (13) in (2), we get

$$
\Gamma_{G}^{\nu}\left(P_{1}, P_{2}\right)=\Gamma_{l}^{\nu}\left(P_{1}, P_{2}\right)+\Gamma_{t}^{\nu}\left(P_{1}, P_{2}\right)
$$

where in the imaginary time formalism

$$
\begin{aligned}
& \Gamma_{l}^{\nu}\left(P_{1}, P_{2}\right)=\frac{4}{3} g^{2} \text { e } i T \sum_{k_{0}} \int \frac{\mathrm{d}^{3} k}{(2 \pi)^{3}} \frac{1}{R^{2} S^{2}}\left[\left(g_{\mu \rho}-\frac{K_{\mu} K_{\rho}}{K^{2}}\right) \gamma^{\mu} \not R \gamma^{\nu} \not \gamma^{\rho}-\gamma^{i} \not R \gamma^{\nu} \not{S} \gamma_{i}\right. \\
& \left.-\gamma^{i} R \gamma^{\nu} \not \gamma^{j} \frac{k_{i} k_{j}}{k^{2}}\right] \tilde{D}_{l}\left(K^{2}\right) \\
& \Gamma_{t}^{\nu}\left(P_{1}, P_{2}\right)=\frac{4}{3} g^{2} e i T \sum_{k_{0}} \int \frac{\mathrm{d}^{3} k}{(2 \pi)^{3}} \frac{1}{R^{2} S^{2}}\left[\gamma^{i} R \gamma^{\nu} \$ \gamma_{i}+\gamma^{i} R \gamma^{\nu} \$ \gamma^{j} \frac{k_{i} k_{j}}{k^{2}}\right] \tilde{D}_{t}\left(K^{2}\right),
\end{aligned}
$$

with $S=P_{1}-K$ and $R=P_{2}+K$. Using again the small $K$ expansion (8) and restricting to the lowest Matsubara mode, $k_{0}=2 \pi i n T=0$, [11] yields

$$
\begin{aligned}
\Gamma_{l}^{\nu}\left(P_{1}, P_{2}\right)= & \frac{i e}{6} g^{2} \frac{1}{P_{1}^{2} P_{2}^{2}}\left[\frac{4}{3} g^{i \nu} \gamma_{i}-\gamma^{\nu}+\gamma^{0} \not P_{2} \gamma^{\nu} \not P_{1} \gamma^{0}\left(\frac{1}{P_{1}^{2}}+\frac{1}{P_{2}^{2}}+\frac{4 p_{1}^{2}}{3 P_{1}^{4}}+\frac{4 p_{2}^{2}}{3 P_{2}^{4}}\right.\right. \\
& \left.-\frac{4\left(\vec{p}_{1} \cdot \vec{p}_{2}\right)}{3 P_{1}^{2} P_{2}^{2}}\right)+\frac{2}{3 P_{1}^{2}} \gamma^{0}\left(\vec{p}_{1} \cdot \vec{\gamma}\right) \gamma^{\nu} \not P_{1} \gamma^{0}-\frac{2}{3 P_{2}^{2}} \gamma^{0}\left(\vec{p}_{2} \cdot \vec{\gamma}\right) \gamma^{\nu} \not P_{1} \gamma^{0} \\
& \left.-\frac{2}{3 P_{1}^{2}} \gamma^{0} \not P_{2}\left(\vec{p}_{1} \cdot \vec{\gamma}\right) \gamma^{0}+\frac{2}{3 P_{2}^{2}} \gamma^{0} \not P_{2} \gamma^{\nu}\left(\vec{p}_{2} \cdot \vec{\gamma}\right) \gamma^{0}\right]\left\langle\mathcal{E}^{2}\right\rangle_{T},
\end{aligned}
$$

and

$$
\begin{aligned}
\Gamma_{t}^{\nu}\left(P_{1}, P_{2}\right)= & -\frac{i e}{12} g^{2} \frac{1}{P_{1}^{2} P_{2}^{2}}\left[2 \gamma^{\nu}-\frac{8}{3} g^{i \nu} \gamma_{i}+\gamma^{i} \not_{2} \gamma^{\nu} \not P_{1} \gamma_{i}\left(\frac{2}{3 P_{1}^{2}}+\frac{2}{3 P_{2}^{2}}+\frac{16 p_{1}^{2}}{15 P_{1}^{4}}+\frac{16 p_{2}^{2}}{15 P_{2}^{4}}\right.\right. \\
& \left.-\frac{16\left(\vec{p}_{1} \cdot \vec{p}_{2}\right)}{15 P_{1}^{2} P_{2}^{2}}\right)-\frac{2}{3 P_{1}^{2}} \gamma^{\nu} \not P_{1}\left(\vec{p}_{1} \cdot \vec{\gamma}\right)+\frac{2}{3 P_{2}^{2}} \gamma^{\nu} \not P_{1}\left(\vec{p}_{2} \cdot \vec{\gamma}\right)+\frac{2}{3 P_{1}^{2}}\left(\vec{p}_{1} \cdot \vec{\gamma}\right) P_{2} \gamma^{\nu} \\
& -\frac{2}{3 P_{2}^{2}}\left(\vec{p}_{2} \cdot \vec{\gamma}\right) \not P_{2} \gamma^{\nu}+\frac{2}{3 P_{1}^{2}} \gamma^{i}\left(\vec{p}_{1} \cdot \vec{\gamma}\right) \gamma^{\nu} P_{1} \gamma_{i}-\frac{2}{3 P_{2}^{2}} \gamma^{i}\left(\vec{p}_{2} \cdot \vec{\gamma}\right) \gamma^{\nu} \not P_{1} \gamma_{i} \\
& -\frac{2}{3 P_{1}^{2}} \gamma^{i} \not P_{2} \gamma^{\nu}\left(\vec{p}_{1} \cdot \vec{\gamma}\right) \gamma_{i}+\frac{2}{3 P_{2}^{2}} \gamma^{i} \not P_{2} \gamma^{\nu}\left(\vec{p}_{2} \cdot \vec{\gamma}\right) \gamma_{i}+\frac{8}{15 P_{1}^{4}}\left(\vec{p}_{1} \cdot \vec{\gamma}\right) P_{2} \gamma^{\nu} \not P_{1}\left(\vec{p}_{1} \cdot \vec{\gamma}\right) \\
& +\frac{8}{15 P_{2}^{4}}\left(\vec{p}_{2} \cdot \vec{\gamma}\right) \not P_{2} \gamma^{\nu} \not P_{1}\left(\vec{p}_{2} \cdot \vec{\gamma}\right)-\frac{4}{15 P_{1}^{2} P_{2}^{2}}\left(\vec{p}_{1} \cdot \vec{\gamma}\right) P_{2} \gamma^{\nu} P_{1}\left(\vec{p}_{2} \cdot \vec{\gamma}\right) \\
& \left.+\frac{4}{15 P_{1}^{2} P_{2}^{2}}\left(\vec{p}_{2} \cdot \vec{\gamma}\right) \not P_{2} \gamma^{\nu} \not P_{1}\left(\vec{p}_{1} \cdot \vec{\gamma}\right)\right]\left\langle\mathcal{B}^{2}\right\rangle_{T} \cdot
\end{aligned}
$$

At finite temperature the moments of the longitudinal and transverse gluon propagator in (12) are related to the in-medium chromoelectric, $\left\langle\mathcal{E}^{2}\right\rangle_{T}$, and chromomagnetic condensates, $\left\langle\mathcal{B}^{2}\right\rangle_{T}$, via 11]

$$
\begin{aligned}
\left\langle\mathcal{E}^{2}\right\rangle_{T} & =\left\langle: G_{a}^{0 i} G_{0 i}^{a}:\right\rangle_{T}=8 T \int \frac{\mathrm{d}^{3} k}{(2 \pi)^{3}} k^{2} \tilde{D}_{l}(0, k), \\
\left\langle\mathcal{B}^{2}\right\rangle_{T} & =\frac{1}{2}\left\langle: G_{a}^{i j} G_{i j}^{a}:\right\rangle_{T}=-16 T \int \frac{\mathrm{d}^{3} k}{(2 \pi)^{3}} k^{2} \tilde{D}_{t}(0, k) .
\end{aligned}
$$


The functions $a$ and $b$ can be expressed in terms of the chromoelectric and chromomagnetic condensates as 11]

$$
\begin{aligned}
& a\left(p_{0}, p\right)=-\frac{g^{2}}{6} \frac{1}{P^{6}}\left[\left(\frac{1}{3} p^{2}-\frac{5}{3} p_{0}^{2}\right)\left\langle\mathcal{E}^{2}\right\rangle_{T}-\left(\frac{1}{5} p^{2}-p_{0}^{2}\right)\left\langle\mathcal{B}^{2}\right\rangle_{T}\right], \\
& b\left(p_{0}, p\right)=-\frac{4}{9} g^{2} \frac{p_{0}}{P^{6}}\left[p_{0}^{2}\left\langle\mathcal{E}^{2}\right\rangle_{T}+\frac{1}{5} p^{2}\left\langle\mathcal{B}^{2}\right\rangle_{T}\right] .
\end{aligned}
$$

Combining (11), (16), (18), and (19) and (44), (14) and (21) one can show again that the effective vertex, obtained above at finite temperature, is related to the effective propagator through the Ward-Takahashi identity

$$
\begin{aligned}
\left(P_{1}+P_{2}\right)_{\nu} \Gamma^{\nu}\left(P_{1}, P_{2}\right) & =-i e\left[\not P_{1}\left(1+a\left(p_{1}^{0}, p_{1}\right)\right)+b\left(p_{1}^{0}, p_{1}\right) \gamma_{0}\right. \\
& \left.-\left(-\not P_{2}\left(1+a\left(-p_{2}^{0}, p_{2}\right)\right)+b\left(-p_{2}^{0}, p_{2}\right) \gamma_{0}\right)\right] \\
& =-i e\left[S^{-1}\left(P_{1}\right)-S^{-1}\left(-P_{2}\right)\right] .
\end{aligned}
$$

It can also be shown that, keeping terms proportional to $k_{0}^{2}$ in (18 21), taking the zero temperature limit, and replacing $i T \sum_{k_{0}} \int \mathrm{d}^{3} k /(2 \pi)^{3} \rightarrow \int \mathrm{d}^{4} k /(2 \pi)^{4}$ along with $\tilde{D}_{L}\left(k_{0}, k\right)=$ $\tilde{D}_{T}\left(k_{0}, k\right)=-\tilde{D}\left(K^{2}\right)$, the zero temperature results for the vertex and the propagator are reproduced.

In Minkowski space the condensates in (20) can be expressed in terms of the space like $\left(\Delta_{\sigma}\right)$ and time like $\left(\Delta_{\tau}\right)$ plaquette expectation values as [11]

$$
\begin{aligned}
\frac{\alpha_{s}}{\pi}\left\langle\mathcal{E}^{2}\right\rangle_{T} & =\frac{4}{11} T^{4} \Delta_{\tau}-\frac{2}{11}\left\langle G^{2}\right\rangle_{T=0}, \\
\frac{\alpha_{s}}{\pi}\left\langle\mathcal{B}^{2}\right\rangle_{T} & =-\frac{4}{11} T^{4} \Delta_{\sigma}+\frac{2}{11}\left\langle G^{2}\right\rangle_{T=0},
\end{aligned}
$$

where $\left\langle G^{2}\right\rangle_{T=0}=(2.5 \pm 1.0) T_{c}^{4}$ [5]. The plaquette expectation values have been measured above the critical temperature $T_{c}$ on the lattice for pure $\mathrm{SU}(3)$ gauge theory [5], and these results can be used to determine the effective vertex and propagator. It should be noted that our results are gauge independent but apply only above the critical temperature. At zero temperature the quark condensate has to be taken into account, which generates serious problems with respect to gauge invariance. The gluon condensate, on the other hand, describing the breaking of the scale invariance exists also in the deconfined phase [16].

The effective quark propagator gives rise to the quark dispersion relations discussed in Ref. [11]. As a possible application of the effective Greens functions, containing nonperturbative effects, they can be used to estimate the dilepton production from a QGP. Recently, this rate has been calculated from the imaginary part of the photon self energy in one-loop approximation using our effective quark propagator [13]. The physical process corresponding to this rate is the annihilation of collective quark-antiquark modes in the QGP. Due to the non-trivial quark dispersion relation interesting structures (peaks, gaps) show up in the rate, in a similar manner as for the corresponding HTL calculation [4]. As in the HTL case also the effective quark-photon vertex, constructed here, should be used in a complete calculation of the one-loop photon self energy. As a matter of fact, in the case of $\pi^{+}-\pi^{-}$annihilation, the consideration of the Ward identity leads to a strong suppression of 
the dilepton production rate [17], whereas the use of an effective HTL vertex results in an enhancement of the QGP rate. Therefore it will be very interesting to see, how the gluon condensate affects the balance of both effects. The practical calculation seems, however, to be rather involved.

We gratefully acknowledge the useful discussion with Stefan Leupold and Ulrich Mosel during the course of this work. 


\section{REFERENCES}

[1] Proceedings of the Fifth International Workshop on Thermal Field Theories and its Applications, Ed. U. Heinz, thep-ph/9811469, Regensburg, August 10-14, 1998.

[2] O. K. Kalashnikov and V. V. Klimov, Sov. J. Nucl. Phys. 31 (1980) 699; V. V. Klimov, Sov. Phys. JETP 55 (1982) 199; H. A. Weldon, Phys. Rev. D 26 (1982) 1394; R. D. Pisarski, Physica 158 (1989) 146; M. H. Thoma, in Quark-Gluon Plasma - II, Ed. R. Hwa (World Scientific, Singapore, 1995) p. 51.

[3] E. Braaten and R. D. Pisarski, Nucl. Phys. B 337 (1990) 569; ibid 339 (1990) 310.

[4] E. Braaten, R.D. Pisarski, and T.C. Yuan, Phys. Rev. Lett. 64, (1990) 2242.

[5] G. Boyd et al., Nucl. Phys. B 469, (1996) 419.

[6] A. Peshier, B. Kämpfer, O.P. Pavlenko, and G. Soff, Phys. Lett. B 337, (1994) 235; A. Peshier, B. Kämpfer, O.P. Pavlenko, and G. Soff, Phys. Rev. D 54, (1996) 2399.

[7] G. Boyd, S. Gupta, F. Karsch, and E. Laermann, Z. Phys. C 64, (1994) 331.

[8] M. A. Shifman, A. I. Vainshtein and V. I. Zakharov, Nucl. Phys. B 147 (1979) 385, $448,519$.

[9] M. J. Lavelle and M. Schaden, Phys. Lett. B 208, (1988) 297.

[10] M. J. Lavelle and M. Schaden, Phys. Lett. B 217, (1989) 297; ibid 246 (1990) 487; M. Lavelle, Phys. Rev. D 44 (1991) R46; E. Bagan et al., Phys. Lett. B 219 (1989) 497; J. Ahlbach et al., Phys. Lett. B 275 (1992) 142.

[11] A. Schäfer and M. H. Thoma, Phys. Lett. B 451, (1999) 195.

[12] I. Schmidt and J.J. Yang, 〈hep-ph/9906510

[13] M. G. Mustafa, A. Schäfer and M. H. Thoma, hep-ph/9906391, To appear in the proceeding of Quark-Matter'99; hep-ph/9908461, Phys. Rev. C (in press).

[14] J. I. Kapusta, Finite Temperature Field Theory (Cambridge University Press, New York, 1989) p. 70.

[15] H. A. Weldon, Phys. Rev. D 26 (1982) 2789.

[16] H. Leutwyler, in Proc. Conf. QCD - 20 years later, Eds. P. M. Zerwas and H. A. Kastrup (World Scientific, Singapore,1993) p. 693.

[17] C. L. Korpa and S. Pratt, Phys. Rev. Lett. 64 (1991) 1502. 


\section{FIGURES}
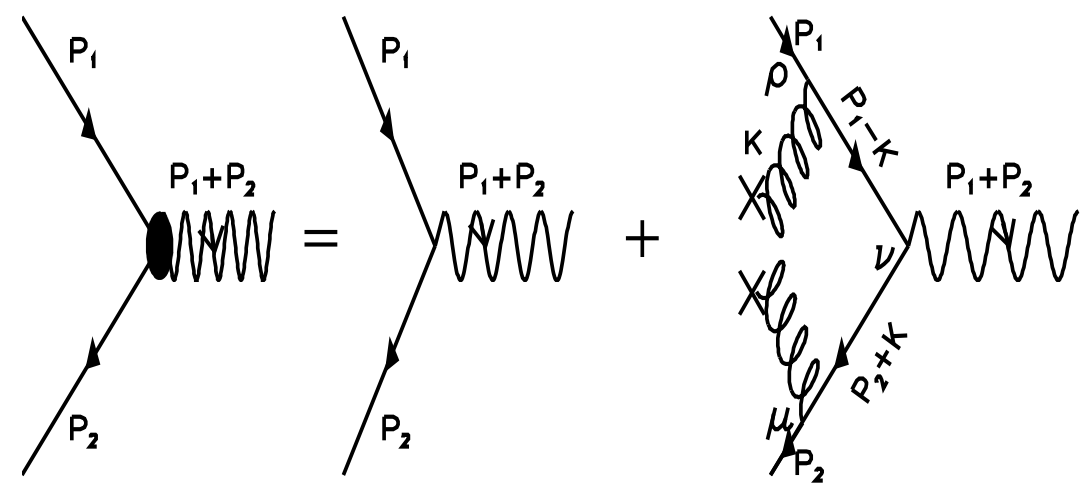

FIG. 1. One-loop correction to the quark-photon vertex containing the gluon condensate.

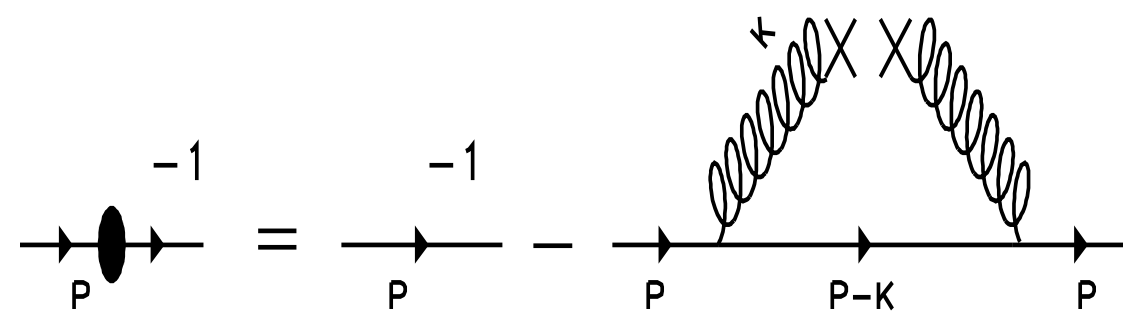

FIG. 2. One-loop correction to the quark propagator containing the gluon condensate. 